Pacific Journal of Mathematics

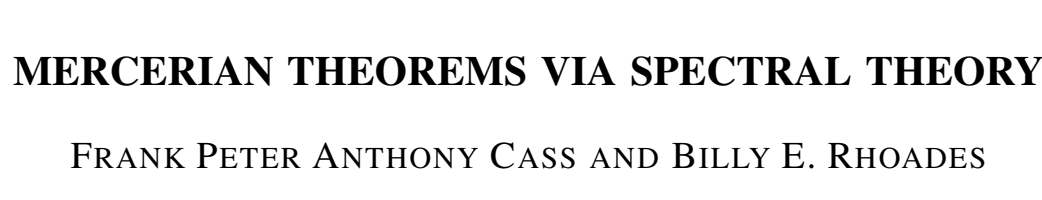




\title{
MERCERIAN THEOREMS VIA SPECTRAL THEORY
}

\author{
Frank P. Cass and B. E. Rhoades
}

Given a regular matrix $A$, Mercerian theorems are concerned with determining the real or complex values of $\alpha$ for which $\alpha I+(1-\alpha) A$ is equivalent to convergence. For $\alpha \neq 1$, the problem is equivalent to determining the resolvent set for $A$, or, determining the spcctrum $\sigma(A)$ of $A$, where $\sigma(A)=$ $\{\lambda \mid A-\lambda I$ is not invertible\}. This paper treats the problem of determining the spectra of weighted mean methods; i.e., triangular matrices $A=\left(a_{n k}\right)$ with $a_{n k}=p_{k} / P_{n}$, where $p_{0}>0$, $p_{n} \geqq 0, \sum_{k=0}^{n} p_{k}=P_{n}$. It is shown that the spectrum of every weighted mean method is contained in the disc $\{\lambda|| \lambda-1 / 2 \mid \leqq$ $1 / 2\}$ (Theorem 1), and, if $\lim p_{n} / P_{n}$ exists,

$$
\begin{aligned}
\sigma(A) & =\left\{\lambda|| \lambda-(2-\varepsilon)^{-1} \mid\right. \\
& \leqq(1-\varepsilon) /(2-\varepsilon)\} \cup\left\{p_{n} / P_{n} \mid p_{n} / P_{n}<\varepsilon /(2-\varepsilon)\right\},
\end{aligned}
$$

where $\varepsilon=\lim p_{n} / P_{n}$.

Let $\gamma=\underline{\lim } p_{n} / P_{n}, \delta=\varlimsup \overline{\lim } p_{n} / P_{n}, S=\left\{\overline{\left.p_{n} / P_{n} \mid n \geqq 0\right\}}\right.$. When $\gamma<\delta$, some examples are provided to indicate the difficulty of determining the spectrum explicitly. It is shown that $\left\{\lambda|| \lambda-(2-\delta)^{-1} \mid \leqq(1-\delta /(2-\delta)\} \cup S \subseteq \sigma(A)\right.$ and

$$
\sigma(A) \leqq\left\{\lambda|| \lambda-(2-\gamma)^{-1} \mid \leqq(1-\gamma) /(2-\gamma)\right\} \cup S .
$$

Theorem 1 is a generalization of the corresponding theorems of: S. Aljancic, L. N. Cakalov, K. Knopp, M. E. Landau, J. Mercer, Y. Okada, W. Sierpinski, and G. Sunouchi.

Using spectral theory we obtain the best possible Mercerian theorems for certain classes of weighted mean methods of summability.

The weighted mean method is a triangular matrix $A=\left(a_{n k}\right)$ with $a_{n k}=p_{k} / P_{n}$, where $p_{0}>0, p_{n} \geqq 0, n \geqq 0, P_{n}=\sum_{k=0}^{n} p_{k}$ and $A$ is a bounded linear operator on $c$, the space of convergent sequences.

For $\alpha \neq 0$ we may write $\alpha I+(1-\alpha) A=\alpha(I+q A)$, where $q=$ $(1-\alpha) / \alpha$. Mercer's original theorem [9] states the following: Let $\left\{x_{n}\right\}$ be a sequence such that $x_{n+1}-x_{n}+\mu n^{-1} x_{n} \rightarrow \lambda$ as $n \rightarrow \infty$. If $\lambda$ is finite and $\mu>-1$, then $x_{n+1}-x_{n}$ and $n^{-1} x_{n}$ both tend to $\lambda /(\mu+1)$ as $n \rightarrow \infty$. (ii) If $\lambda$ is infinite and $\mu>-1$, then $n^{-1} x_{n} \rightarrow \lambda$ and $x_{n+1}-x_{n} \rightarrow \lambda$ only if $0 \geqq \mu>-1$.

Landau [8] showed that, if $\left\{x_{n}\right\}$ is a complex sequence, $q$ a positive integer, then $\lim _{n}\left(x_{n}+(q / n) \sum_{k=1}^{n} x_{k}\right)=0$ implies $\lim _{n} x_{n}=0$. Sierpinski [14] extended Landau's result to real numbers $q>-1$ and showed it could not be extended to $q \leqq-1$. Sierpinski's result for $q>-1$ was reproved in [3]. 
Let $\sum_{n=2}^{\infty} p_{n} /\left(p_{1}+p_{2}+\cdots+p_{n-1}\right)$ be a divergent series of positive terms, $\left\{x_{n}\right\}$ a complex sequence. Okada [10] showed that if $q>-1$, then $\lim _{n}\left(x_{n}+q\left(\sum_{k=1}^{n} p_{k} x_{k} / \sum_{k=1}^{n} p_{k}\right)\right)=l, l$ finite, implies $\lim _{n} x_{n}=l /(1+q)$. He also verified that the theorem does not hold for $\underline{\lim }_{n} \sum_{k=1}^{n-1} p_{k} / p_{n}>$ $-(1+q) \geqq 0$.

Using a different technique, Knopp [6] reproved Okada's result. Beekman [2] showed that, if $A$ is a conservative triangle with inverse satisfying $a_{n n}^{-1}>0, a_{n k}^{-1} \leqq 0$ for $n>k$, then $I+q A$ is equivalent to convergence for $\operatorname{Re}(q)>-1$.

We determine the spectrum of $A, \sigma(A)$, in every case in which $\lim p_{n} / P_{n}$ exists (Corollaries 1 and 2). When $\left\{p_{n} / P_{n}\right\}$ does not converge, in which case $A$ is necessarily regular, the situation seems pathological: Theorems 2 and 3 do give set inclusions for $\sigma(A)$, but, as we show by examples, $\sigma(A)$ can be disconnected and is very difficult to describe explicitly.

Let $B=A-\lambda I$. Our first task is to compute the entries of $B^{-1}$. Except for Theorem 1, we shall restrict our attention to regular weighted mean methods; i.e., those for which $P_{n} \rightarrow \infty$. For, if $P_{n}$ tends to a finite limit, then $A$ is compact and $\sigma(A)=\left\{p_{k} / P_{k}: k \geqq 0\right\} \cup\{0\}$. (See, e.g. [13, Theorem 1].)

Lemma 1. Let $A$ be a weighted mean matrix, $B=A-\lambda I, \lambda a$ scalar such that $b_{n n} \neq 0$ for each $n$. Then $D=B^{-1}$ has entries

$$
\begin{aligned}
d_{n n}= & \frac{P_{n}}{p_{n}-\lambda P_{n}}, \\
& d_{n k}=(-1)^{n+k} \frac{\lambda^{n-k-1} p_{k}}{P_{n}} \prod_{j=k}^{n} \frac{P_{j}}{p_{j}-\lambda P_{j}}, \quad k<n .
\end{aligned}
$$

Proof. A direct computation verifies $d_{n n}$ and $d_{n, n-1}$. By induction one can show that

$$
\sum_{j=0}^{k}(-1)^{j} \lambda^{j-1} \frac{p_{n-j}}{P_{n-j}} \prod_{i=0}^{j} \frac{P_{n-i}}{p_{n-i}-\lambda P_{n-i}}=(-1)^{k} \lambda^{k} \prod_{j=0}^{k} \frac{P_{n-j}}{P_{n-j}-\lambda P_{n-j}} .
$$

With (2), one verifies by induction that (1) is true.

Theorem 1. Let $A$ be a weighted mean method. Then $\sigma(A) \subseteq$ $\{z|| z-1 / 2 \mid \leqq 1 / 2\}$.

Proof. Let $\lambda=x+i y$ satisfy $|\lambda-1 / 2|>1 / 2$. This inequality is equivalent to $\alpha>-1$, where $-1 / \lambda=\alpha+i \beta$. Since $\alpha>-1$ and $0 \leqq p_{j} / P_{j} \leqq 1$ for all $j,\left|1-p_{j} / \lambda P_{j}\right| \geqq \mid 1+\alpha p_{j} / P_{j}=1+\alpha p_{j} / P_{j}$. For $k<n,\left|d_{n k}\right| \leqq p_{k} /|\lambda|^{2} P_{n} \Pi_{j=k}^{n}\left(1+\alpha p_{j} / P_{j}\right)=f_{n k}$, say.

Using finite induction we can show, for each $0<r<n$, 


$$
\sum_{k=0}^{r} f_{n k}=\frac{P_{r}}{|\lambda|^{2} P_{n}(1+\alpha) \prod_{j=r+1}^{n}\left(1+\alpha p_{j} / P_{j}\right)} .
$$

Therefore $\sum_{k=0}^{n}\left|d_{n n}\right| \leqq\left|d_{n n}\right|+\sum_{k=0}^{n-1} f_{n k}=\left|d_{n n}\right|+P_{n-1} /|\lambda|^{2} P_{n}$.

$$
\begin{aligned}
(1+\alpha)\left(1+\alpha p_{n} / P_{n}\right) & \leqq\left|p_{n} / P_{n}-\lambda\right|^{-1}+\beta|\lambda|^{-2}(1+\alpha)^{-1} \\
& \leqq \beta|\lambda|^{-1}(1+1 /|\lambda|(1+\alpha)),
\end{aligned}
$$

where $\beta=1$ if $\alpha \geqq 0$ and $\beta=(1+\alpha)^{-1}$ if $-1<\alpha<0$. Since $d_{n n} \neq$ 0 for each $n$, from Problem 32 [16, p. 232], the convergence domain of $D,(D)$, is equal to $c$, and $\lambda \in \rho(A)$, the resolvent of $A$.

Theorem 1 is a special case of [2, Theorem 1]. Since 0 is not an interior point of $\sigma(A)$, Theorem 1 provides another proof of the fact that every weighted mean method lies in the closure of the maximal group of invertible elements in 4 , the subalgebra of $B(c)$ consisting of triangular matrices. (See [11, p. 287].)

Let $\delta=\varlimsup_{n} p_{n} / P_{n}, \gamma=\underline{\lim }_{n} p_{n} / P_{n}$.

THeORem 2. Let $A$ be a regular weighted mean method. Then $\sigma(A) \supseteqq\left\{\lambda|| \lambda-(2-\delta)^{-1} \mid \leqq(1-\delta) /(2-\delta)\right\} \cup S$, where $S=\overline{\left\{p_{n} / P_{n} \mid n \geqq 0\right\}}$.

Proof. Fix $\lambda$ satisfying $\left|\lambda-(2-\delta)^{-1}\right|<(1-\delta) /(2-\delta)$ and $\lambda \neq$ $p_{n} / P_{n}$ for any $n$. From (1) we obtain

$$
\left|d_{n k}\right|=\frac{p_{k}}{|\lambda|^{2} P_{k-1} \prod_{j=k}^{n}\left|1+\left(1-\frac{1}{\lambda}\right) \frac{p_{j}}{P_{j-1}}\right|} .
$$

Note that $\mid 1+\left(1-(1 / \lambda) p_{n+1} / P_{n} \mid \leqq 1\right.$ if and only if

$$
\left(1+(1+\alpha) p_{n+1} / P_{n}\right)^{2}+\left(\beta p_{n+1} / P_{n}\right)^{2} \leqq 1,
$$

where $-1 / \lambda=\alpha+i \beta$; i.e.,

$$
2(1+\alpha) p_{n+1} / P_{n}+\left((1+\alpha)^{2}+\beta^{2}\right)\left(p_{n+1} / P_{n}\right)^{2}<0 .
$$

For each $n$ such that $p_{n+1}=0,(4)$ is automatically satisfied. For each $n$ such that $p_{n+1}>0,(4)$ is equivalent to

$$
2(1+\alpha)+\left((1+\alpha)^{2}+\beta^{2}\right) p_{n+1} / P_{n} \leqq 0 .
$$

For (5) to be true for all $n$ sufficiently large, it is sufficient to have $\delta$ satisfy

$$
2(1+\alpha)+\left((1+\alpha)^{2}+\beta^{2}\right) \delta /(1-\delta)<0,
$$

since $p_{n+1} / P_{n}=p_{n+1} / P_{n+1}\left(1-p_{n+1} / P_{n+1}\right)$, which is monotone increasing in $p_{n} / P_{n}$. Inequality (6) is equivalent to $\left|\lambda-(2-\delta)^{-1}\right|<(1-\delta) /(2-\delta)$. 
Therefore, for all $n \geqq N$, using (3),

$$
\sum_{k=N}^{n-1}\left|d_{n k}\right| \geqq \frac{1}{|\lambda|^{2}} \sum_{k=N}^{n-1} \frac{p_{k}}{P_{k-1}} \geqq \frac{1}{|\lambda|^{2}} \sum_{k=N}^{n-1} \frac{p_{k}}{P_{k}},
$$

which diverges by the Abel-Dini theorem [7, p. 290].

If $\lambda=p_{n} / P_{n}$ then $\lambda$ belongs to the spectrum of $A$. Theorem 2 follows since the spectrum is always closed.

CoROLLARY 1. Let $A$ be a regular weighted mean method with $\delta=0$. Then $\sigma(A)=\{\lambda|| \lambda-1 / 2 \mid \leqq 1 / 2\}$.

Proof. Combine Theorems 1 and 2, observing that $S$ is already contained in the disc.

Special cases of Corollary 1 for $\lambda$ real appear in [1], [6], and [10].

THEOREM 3. Let $A$ be a regular weighted mean method with $\gamma>0$. Then $\sigma(A) \subseteq\left\{\lambda|| \lambda-(2-\gamma)^{-1} \mid<(1-\lambda) /(2-\gamma)\right\} \cup S$.

Proof. Let $\lambda$ be fixed and satisfy $\left|\lambda-(2-\gamma)^{-1}\right|>(1-\gamma) /(2-\gamma)$ and $\lambda \neq p_{n} / P_{n}$ for any $n$. We shall show that $\lambda \in \rho(A)$, the resolvent of $A$. From Theorem 1 we need consider only those values of $\lambda$ satisfying $|\lambda-1 / 2| \leqq 1 / 2$; i.e., $\alpha<-1$. The value $\alpha=-1$ corresponds to $\lambda=1$, which we know lies in the spectrum, since $p_{0} / P_{0}=1$. Therefore we shall assume $\alpha<-1$.

Under the assumption on $\lambda$ we wish to verify that

$$
\left|1+(1-1 / \lambda) p_{j} / P_{j-1}\right|
$$

is strictly larger than one for all $j$ sufficiently large. To this end, define $f(t)=1+2(1+\alpha) t+\left((1+\alpha)^{2}+\beta^{2}\right) t^{2} . \quad f$ has a minimum at $t_{0}=-(1+\alpha) /\left((1+\alpha)^{2}+\beta^{2}\right)$.

The assumption on $\lambda$ is equivalent to

$$
\gamma\left(\alpha^{2}+\beta^{2}\right)+2 \alpha>\gamma-2 \text {. }
$$

Therefore

$$
\frac{\gamma}{2(1-\gamma)}>\frac{-(1+\alpha)}{(1+\alpha)^{2}+\beta^{2}}=t_{0}
$$

and $f$ is monotone increasing for all $t>\gamma / 2(1-\gamma)$.

Let $\varepsilon>0$ and small. $f((\gamma /(1-\gamma))-\varepsilon)=f(\gamma /(1-\gamma))-2 \in g(\varepsilon)$, where $g(\varepsilon)=1+\alpha+\left((1+\alpha)^{2}+\beta^{2}\right)(\gamma /(1-\gamma)-\varepsilon / 2) . \quad g(\varepsilon)>0$ for small $\varepsilon$, since $f$ is monotone increasing for $t>\gamma / 2(1-\gamma)$.

We shall now show that $f(\gamma /(1-\gamma))>1$. From the hypothesis on $\lambda$ and (6), 


$$
\alpha^{2}+\beta^{2}+\frac{2 \alpha}{\gamma}>\frac{\gamma-2}{\gamma}
$$

which is equivalent to

$$
\left|\frac{1}{1-\gamma}-\frac{\gamma}{\lambda(1-\lambda)}\right|>1
$$

But $1 /(1-\gamma)=1+\gamma /(1-\gamma)$, so we have

$$
\left(f(\gamma /(1-\gamma))=|1+(1-1 / \lambda) \gamma /(1-\gamma)|^{2}>1 .\right.
$$

Now choose $\varepsilon>0$ and so small that $f(\gamma /(1-\gamma)-\varepsilon)=f(\gamma /(1-\gamma))-$ $2 \varepsilon g(\varepsilon)=m^{2}>1$. Then, by the definition of $\gamma$ there exists an $N$ such that $n>N$ implies $p_{n+1} / P_{n}>\gamma /(1-\gamma)-\varepsilon$, so that $f\left(p_{n} / P_{n-1}\right)>$ $f(\gamma /(1-\gamma)-\varepsilon)=m^{2}$.

Using (3), $\left|d_{n k}\right| /\left|d_{n+1, k}\right|=\left(f\left(p_{n+1} / P_{n}\right)\right)>m^{2}>1$ for all $n \geqq N$. Therefore $\left|d_{n k}\right|$ is monotone decreasing in $n$ for each $k, n \geqq N$, so that $D$ has bounded columns. Thus, to show that $D$ has finite norm it is sufficient to show that $\left|d_{n n}\right|$ is bounded, and that $\sum_{k=N}^{n-1}\left|d_{n k}\right|$ is bounded.

Recall that $p_{n} / P_{n-1}$ is monotone increasing in $p_{n} / P_{n}$. For the $\varepsilon$ we are using, we can enlarge $N$, if necessary, to ensure that $p_{n} / P_{n-1}<$ $\delta /(1-\delta)+1$ for $n \geqq N$.

From (3),

$$
\begin{aligned}
\sum_{k=N}^{n-1}|| d_{n k} \mid & \leqq \frac{1}{|\lambda|^{2}}\left(\frac{\delta}{1-\delta}+1\right) \sum_{k=N}^{n-1}\left(\prod_{j=k}^{n}\left|1+\left(1-\frac{1}{\lambda}\right) \frac{p_{j}}{P_{j-1}}\right|^{-1}\right. \\
& \leqq \frac{1}{|\lambda|^{2}}\left(\frac{\delta}{1-\delta}+1\right) \sum_{k=N}^{n-1} m^{-n+k-1}<H
\end{aligned}
$$

where $H$ is independent of $n$.

$$
\begin{aligned}
\left|d_{n n}\right| & =\frac{P_{n}}{\left|p_{n}-\lambda P_{n}\right|}=\frac{P_{n}}{|\lambda|\left|P_{n}-p_{n} / \lambda\right|}=\frac{P_{n}}{|\lambda|\left|P_{n-1}+(1-1 / \lambda) p_{n}\right|} \\
& =\frac{P_{n} / P_{n-1}}{|\lambda|\left|1+(1-1 / \lambda) p_{n} / P_{n-1}\right|}=\frac{\left(1+p_{n} / P_{n-1}\right)}{|\lambda|\left|1+(1-1 / \lambda) p_{n} / P_{n-1}\right|} \\
& <\frac{1+\delta /(1-\delta)+1}{|\lambda| m} .
\end{aligned}
$$

Therefore $D$ has finite norm. From [16, loc. cit.], $(D)=c$ and $\lambda \epsilon$ $\rho(A)$.

CoROLLARY 2. Let $A$ be a regular weighted mean method with $\lim _{n} p_{n} / P_{n}=\gamma>0$. Then $\sigma(A)=\left\{\lambda \| \lambda-(2-\gamma)^{-1} \mid \leqq(1-\gamma) /(2-\gamma)\right\} \cup$ $E$, where $E=\left\{p_{n} / P_{n} \mid p_{n} / P_{n}<\gamma /(2-\gamma)\right\}$. 
Proof. Combine Theorems 2 and 3 and note that $S \backslash E$ is already contained in the disc, and $E$ is a finite set.

We now obtain a necessary and sufficient condition for a weighted mean method to be equivalent to convergence.

THeOREM 4. Let $A$ be a regular weighted mean method. Then $(A)=c$ if and only if $\theta=\underline{\lim }_{n} p_{n+1} / P_{n}>0$.

Proof. $\theta>0$ implies $p_{n+1} / P_{n} \geqq \theta / 2$ for all $n$ sufficiently large. For each $n p_{n+1} / P_{n+1}=\left(p_{n+1} / P_{n}\right) /\left(1+p_{n+1} / P_{n}\right)$. Note that $f(y)=y /(1+y)$ is monotone increasing in $y$, so that, for all $n \geqq N, p_{n+1} / P_{n+1} \geqq \theta /(2+\theta)$, and the diagonal entries of $A$ are nonzero for $n \geqq N$. If $a_{n n}=0$ for any $n<N$, replace the zero by 1 . The new matrix $B$ has the same convergence domain as $A$. For $n \geqq N$, the nonzero terms of $B^{-1}$ are $b_{n n}^{-1}=P_{n} / p_{n}, b_{n, n-1}^{-1}=-P_{n-1} / p_{n}$.

Suppose $a_{k k}=0$ for some $k<N$. Then $p_{k}=0, b_{k k}=1$ and $b_{n k}=0$ for $n>k$. Thus $b_{k k}^{-1}=1, b_{k+1, k}^{-1}=0$ and, by induction, $b_{n k}^{-1}=0$ for $n>k$.

Therefore $\left\|B^{-1}\right\|=\sup _{n}\left[P_{n-1} / p_{n}+P_{n} / p_{n}\right] \leqq \sup _{n} 2 P_{n} / p_{n} \leqq 2(2+\theta) / \theta<$ $\infty$. By $[16],(B)=c$. Thus $(A)=c$.

Suppose $\theta=0$. Then there exists a subsequence $\left\{n_{k}\right\}$ of $n$ such that $\lim _{k} p_{n_{k}+1} / P_{n_{k}}=0$.

Case I. $p_{n}=0$ for at most a finite number of values of $n$. Let $B$ be the matrix $A$ with each zero diagonal entry replaced by 1 . Then $(B)=(A)$. Since $p_{n+1} / P_{n+1}=\left(p_{n+1} / P_{n}\right) /\left(1+p_{n+1} / P_{n}\right), \lim _{k} P_{n_{k}} / p_{n_{k}}=0$. Therefore $\| B^{-1}|| \geqq \sup _{k}\left|b_{n_{k}, n_{k}}^{-1}\right|=+\infty$, and $(B) \neq c$.

Case II. $p_{n}=0$ for an infinite number of values of $n$. Let $\left\{n_{k}\right\}$ denote this set. Define a sequence $\left\{x_{n}\right\}$ by $x_{n_{k}}=1, x_{k}=0$ otherwise. Then $A x=0$, and $(A) \neq c$.

The special case of this theorem for $0<p_{n} \leqq 1$ appears in [4]. A special case of the sufficiency of this theorem appears in [5, p. 59].

We now consider the pathology which may arise when $\gamma<\delta$.

With $p_{0}=1, p_{n} \geqq 0$ for $n>0, c_{n}=p_{n} / P_{n}$, then, as in [12, pp. 163-4], one can show that $p_{n}=c_{n} \amalg_{j=1}^{n}\left(1-c_{j}\right)^{-1}, c_{0}=1,0 \leqq c_{n}<1$ for $n>0$, and $P_{n} \rightarrow \infty$ is equivalent to $\sum_{n=0}^{\infty} c_{n}=\infty$.

For any sequence $s=\left\{s_{n}\right\}$ define $u_{n}=\sum_{k=0}^{n} p_{k} s_{k} / P_{n}$. Then $u_{n}-$ $\left(1-c_{n}\right) u_{n-1}=c_{n} s_{n}$. Let

$$
t_{n}=u_{n}-\lambda s_{n}
$$


For each $c_{n} \neq 0$,

$$
t_{n}=\lambda\left(1-c_{n}\right) u_{n-1} / c_{n}+\left(1-\lambda / c_{n}\right) u_{n} .
$$

Now for the examples. Let $p, q$ be real numbers satisfying $1<$ $p<q$. Define $\left\{c_{n}\right\}$ by $c_{0}=1, c_{2 n}=1 / p, c_{2 n-1}=1 / q, n>0$. Using (8) and (9), $t_{0}=(1-\lambda) u_{0}, t_{2 n}=(p-1) \lambda u_{2 n-1}+(1-p \lambda) u_{2 n}$, and $t_{2 n+1}=$ $(q-1) \lambda u_{2 n}+(1-q \lambda) u_{2 n+1}$. Therefore $t=B u$, where $b_{00}=1, b_{2 n, 2 n}=$ $1-p \lambda, b_{2 n-1,2 n-1}=1-q \lambda, b_{2 n, 2 n-1}=(q-1) \lambda, b_{2 n-1,2 n-2}=(p-1) \lambda, n>0$, $b_{n k}=0$ otherwise. From Theorem $4,(A)=c$.

Suppose $\lambda \neq\{1 / p, 1 / q, 1\}$, and let $E=B^{-1}$. If $\|E\|<\infty$, then from [16, loc. cit.] $E$ is conservative and $(B)=c$. Therefore $t \in c \Rightarrow$ $u \in c \Rightarrow s \in c$ and $(A-\lambda I)=c$, which implies $\lambda \notin \sigma(A)$. Conversely, if $\lambda \notin \sigma(A)$, then $(A-\lambda I)=c$, so that $t \in c \Rightarrow s \in c \Rightarrow u \in c \Rightarrow E$ is conservative $\Rightarrow\|E\|<\infty$. We have shown that, if $\lambda \neq\{1 / p, 1 / q, 1\}$ then $\lambda \notin \sigma(A)$ if and only if $\|E\|<\infty$.

To compute the norm of $E$, observe that $b_{n n} e_{n k}+b_{n, n-1} e_{n-1, k}=0$ for $k<n$, so that $e_{n k}=-b_{n, n-1} e_{n-1, k} / b_{n n}$.

Thus $e_{2 n, k}=-(p-1) \lambda e_{2 n-1, k} /(1-p \lambda), k<2 n, n=1,2, \cdots$, and $e_{2 n+1, k}=-(q-1) \lambda e_{2 n, k} /(1-q \lambda)$. Let $R_{n}=\sum_{k=0}^{n}\left|e_{n k}\right|$. For $n \geqq 1$,

$$
\begin{aligned}
R_{2 n} & =\sum_{k=0}^{2 n-1}\left|e_{2 n, k}\right|+\left|e_{2 n, 2 n}\right| \\
& =\frac{(p-1)|\lambda|}{|1-p \lambda|} \sum_{k=0}^{2 n-1}\left|e_{2 n-1, k}\right|+\frac{1}{|1-p \lambda|} \\
& =\frac{1}{|1-p \lambda|}\left[(p-1)|\lambda| R_{2 n-1}+1\right],
\end{aligned}
$$

and, for $n \geqq 0$,

$$
R_{2 n+1}=\frac{1}{|1-q \lambda|}\left[(q-1)|\lambda| R_{2 n}+1\right] .
$$

Substituting (11) into (10) we have

$$
R_{2 n+2}=\frac{(p-1)(q-1)|\lambda|^{2}}{|1-p \lambda||1-q \lambda|} R_{2 n}+\frac{(p-1)|\lambda|}{|1-p \lambda||1-q \lambda|}+\frac{1}{|1-p \lambda|},
$$

and

$$
R_{2 n+1}=\frac{(p-1)(q-1)|\lambda|^{2}}{|1-p \lambda||1-q \lambda|} R_{2 n-1}+\frac{(q-1)|\lambda|}{|1-p \lambda||1-q \lambda|}+\frac{1}{|1-q \lambda|} .
$$

Let $\left\{\sigma_{n}\right\}$ be defined by $\sigma_{n+1}=\alpha \sigma_{n}+b$, where $a$ and $b$ are fixed positive constants. Then 


$$
\frac{\sigma_{n+1}}{a^{n+1}}-\frac{\sigma_{n}}{a^{n}}=\frac{b}{a^{n+1}},
$$

so that

$$
\frac{\sigma_{n+1}}{a^{n+1}}-\frac{\sigma_{0}}{a^{0}}=\frac{b}{a} \frac{\left(1-a^{-n-1}\right)}{\left(1-a^{-1}\right)}
$$

or $\sigma_{n+1}-\sigma_{0} a^{n+1}=b\left(a^{n+1}-1\right) /(a-1)$. For $0<a<1,\left\{\sigma_{n}\right\}$ is bounded, and, for $a \geqq 1,\left\{\sigma_{n}\right\}$ is unbounded. Therefore

$$
\begin{aligned}
\sigma(A) & =\{\lambda \mid\|E\|=\infty\} \cup\{1 / p, 1 / q, 1\} \\
& =\left\{\left.\lambda|(p-1)(q-1)| \lambda\right|^{2} \geqq|1-p \lambda||1-q \lambda|\right\},
\end{aligned}
$$

since $1 / p, 1 / q$ and 1 already belong to those values of $\lambda$ for which $\|E\|=\infty$.

For $p=2, q=3, \partial \sigma(A)$ is an oval with $x$-intercepts of $1 / 4,1$. For $p=2, q=8$, the boundary consists of a pair of ovals which are tangent at $x=(10-\sqrt{8}) / 23$. For $p=3, q=13, \sigma(A)$ is contained in two disjoint ovals. The left oval has $x$-intercepts at $1 / 15,1 / 9$, and the right oval has $x$-intercepts at $1 / 7,1$.

\section{REFERENCES}

1. S. Aljančić, Sur le Theorème Mercerien de Čakalov, Publ. L'Inst. Math. (Beograd), 19, (1975) 9-15.

2. W. Beekman, Mercer-Sätze für abschnittsbeschrankte Matrix transformationen, Math. Z., 97, (1967), 154-157.

3. L. N. Čakalov, Generalization of a theorem of Mercer on convergence, Izv. Mat. Inst. Acad. Bulgar. Sci., 1., (1954), 85-88.

4. C. W. Groetsch, Summation methods associated with an iteration, Nanta Math., 7 (1974) 13-16.

5. G. H. Hardy, Divergent Series, Oxford University Press, 1949.

6. K. Knopp, Zur Theorie der C-und-H-Summierbarkeit, Math. Z., 19 (1923) 97-113.

7. - Theory and Application of Infinite Series, Blackie and Son Ltd., London, 1947.

8. M. E. Landau, Darstellung und Begründing einiger neurer Ergebnisse der Funktiontheorie, Berlin, (1916), 30.

9. J. Mercer, On the limit of real variants, Proc. London Math. Soc., (2) 5 (1907), 206-224.

10. Y. Okada, A theorem on limits, Tôhoku Math. J., 15 (1919), 280-283.

11. B. E. Rhoades, Triangular summability methods and the boundary of the maximal group, Math. Z., 105 (1968), 284-290.

12. - Fixed point iterations using infinite matrices, Trans. Amer. Math. Soc., 196 (1974), 161-176.

13. N. K. Sharma, Spectra of conservative matrices, Proc. Amer. Math. Soc., 35 (1972), 515-518.

14. W. Sierpinski, Sur la dependance entre l'existence de limites des suites $x_{n}+$ $q\left(x_{1}+x_{2}+\cdot+x_{n}\right) / n$ et $x_{n}$, Tôhoku Math. J., 11 (1914), 1-14.

15. G. Sunouchi, Notes on Fourier analysis (xviii), Absolute summability of series with constant terms, Tôhoku Math. J., (2) 1 (1949-50), 57-65. 
16. A. Wilansky, Functional Analysis, Blaisdell, 1964.

Received March 29, 1977. Preparation of this paper was partially supported by Grant No. A 4806 from the National Research Council of Canada.

UNIVERSITY OF WESTERN ONTARIO

LONDON, ONTARIO

CANADA

AND

INDIANA UNIVERSITY

BLOOMINGTON, IN 47401 



\section{PACIFIC JOURNAL OF MATHEMATICS}

EDITORS

RICHARD ARENS (Managing Editor)

University of California

Los Angeles, California 90024

C. W. Curtis

University of Oregon

Eugene, OR 97403

C. C. MOORE

J. DUGUNDJI

Department of Mathematics University of Southern Californa Los Angeles, California 90007

R. Finn AND J. Milgram Stanford University Stanford, California 94305

University of California

Berkeley, CA 94720

\section{ASSOCIATE EDITORS}

E. F. BeCKENBACH

B. H. NeUMANN

F. WOLF

K. YosHida

\section{SUPPORTING INSTITUTIONS}

UNIVERSITY OF BRITISH COLUMBIA UNIVERSITY OF SOUTHERN CALIFORNIA CALIFORNIA INSTITUTE OF TECHNOLOGY UNIVERSITY OF CALIFORNIA MONTANA STATE UNIVERSITY UNIVERSITY OF NEVADA, RENO STANFORD UNIVERSITY UNIVERSITY OF TOKYO UNIVERSITY OF UTAH NEW MEXICO STATE UNIVERSITY WASHINGTON STATE UNIVERSITY OREGON STATE UNIVERSITY UNIVERSITY OF WASHINGTON UNIVERSITY OF OREGON OSAKA UNIVERSITY 


\section{Pacific Journal of Mathematics \\ Vol. 73, No. 1 \\ March, 1977}

Thomas Robert Berger, Hall-Higman type theorems. $V \ldots \ldots \ldots \ldots \ldots \ldots \ldots$

Frank Peter Anthony Cass and Billy E. Rhoades, Mercerian theorems via

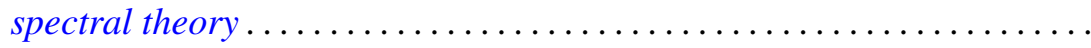

Morris Leroy Eaton and Michael David Perlman, Generating $\mathrm{O}(n)$ with

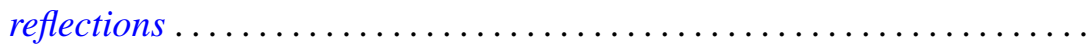

Frank John Forelli, Jr., A necessary condition on the extreme points of a

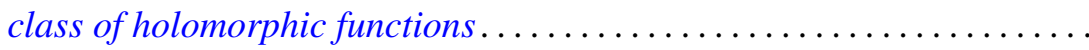

Melvin F. Janowitz, Complemented congruences on complemented

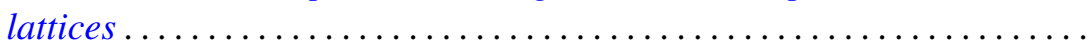

Maria M. Klawe, Semidirect product of semigroups in relation to amenability, cancellation properties, and strong $F \phi$ lner conditions....

Theodore Willis Laetsch, Normal cones, barrier cones, and the "spherical image" of convex surfaces in locally convex spaces ................

Chao-Chu Liang, Involutions fixing codimension two knots.............

Joyce Longman, On generalizations of alternative algebras .............

Giancarlo Mauceri, Square integrable representations and the Fourier

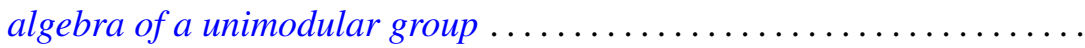

J. Marshall Osborn, Lie algebras with descending chain condition...

John Robert Quine, Jr., Tangent winding numbers and branched

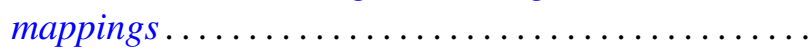

Louis Jackson Ratliff, Jr. and David Eugene Rush, Notes on ideal covers and associated primes .

H. B. Reiter and N. Stavrakas, On the compactness of the hyperspace of faces.

Walter Roth, A general Rudin-Carlson theorem in Banach-spaces ..

Mark Andrew Smith, Products of Banach spaces that are uniformly rotund in every direction.

Roger R. Smith, The R-Borel structure on a Choquet simplex ...

Gerald Stoller, The convergence-preserving rearrangements of real infinite series. ...

Graham H. Toomer, Generalized homotopy excision theorems modulo a Serre class of nilpotent groups...

Norris Freeman Weaver, Dehn's construction and the Poincaré conjecture....

Steven Howard Weintraub, Topological realization of equivariant intersection forms... 